Original Article

\title{
KNOWLEDGE AND PRACTICES REGARDING VITAMIN D DEFICIENCY AMONG WOMEN ATTENDING IMAM HUSSEIN MEDICAL CITY IN KARBALA 2018
}

\author{
SARAH JAFAR AL-GHRAIBAWI ${ }^{1 *}$, SAAD IBRAHIM AL-GHABBAN ${ }^{2}$, RIYADH DHEYHOOD AL-ZUBAIDY2 \\ ${ }^{1}$ Karbala Health Directorate/Primary Health Centers, Karbala, Iraq, ${ }^{2}$ Kerbala University/Collage of Medicine, Karbala, Iraq \\ Email: daf8877@gmail.com
}

Received: 13 Aug 2019, Revised and Accepted: 10 Oct 2019

\section{ABSTRACT}

Objective: Vitamin D deficiency is a global health problem. It is significantly associated with skeletal and non-skeletal problems. Prevention can be maintained mainly by effective sun exposure or supplementation and to lesser extent by dietary intake. To determine the magnitude of knowledge and practice of women towards vitamin D deficiency.

Methods: A cross-sectional study, purposive sample of 442 women were approached who attended consultants' clinics of Imam Hussein Medical City in Karbala. Data collection done between March 2 and June 30, 2018 through direct interviews using a special questionnaire prepared for the purpose of the study.

Results: Less than half (45.2\%) of the women had heard about vitamin D; their source of information was from relatives and friends then from health care provider. The mean score for the participants' knowledge about VDD was 4.25 out of 9.0 (47\%). It has a significant association with higher educational levels and urban residence. There is no significant difference regarding age, marital status or occupation. The mean score for the participants' practice about vitamin D deficiency was 2.53 out of 5 (50.71\%). There is no influence of practice score by socio-demographic factors.

Conclusion: Overall knowledge towards vitamin D deficiency was suboptimal. Improving practices towards VD is directly related to increase knowledge about it that indicates the need to increase public awareness about the problem, encourage appropriate way of sun exposure, consumption of vitamin D rich food and taking supplementation when sun exposure is difficult.

Keywords: Vitamin D Deficiency, Vitamin D, Knowledge, Practices

(C) 2019 The Authors. Published by Innovare Academic Sciences Pvt Ltd. This is an open-access article under the CC BY license (http://creativecommons.org/licenses/by/4.0/) DOI: http://dx.doi.org/10.22159/ijcpr.2019v11i6.36339

\section{INTRODUCTION}

Nowadays the whole world is complaining of an unhealthy lifestyle and incorrect habits that can lead to several disorders like Vitamin D Deficiency (VDD), which affecting about 1 billion people worldwide [1]. The Endocrine Society had been defined VDD as a serum circulating 25-hydroxyvitamin D level $(25(\mathrm{OH}) \mathrm{D})<20 \mathrm{ng} / \mathrm{ml}$, and Vitamin D insufficiency as a 25(OH)D of 21-29 ng/ml [2]. Vitamin D (VD) can be provided through Sun exposure, dietary sources and supplements [3]. In humans, It is thought that about 10,000 units of VD are produced within 30 min of full-body exposure to the sun. It is believed that approximately 5-30 min of exposure to sunlight between $10 \mathrm{a}$. m. to $3 \mathrm{p}$. m. in most human-populated latitudes, at least twice a week, to the face, arms, legs, or back without using sunscreen can produce the required amount of VD [4]. Vitamin D can be obtained from various types of foods in small amount and from fortified foods and supplements in more extent amount [5]. The dependence on dietary sources to provide adequate intakes of VD especially in vulnerable groups during winter month is increased If sunlight exposure is limited [6]. Vitamin D is responsible for a lot of functions in the human body [7]. It has an important function in variant metabolic processes and neuromuscular activities [8]. Clinical presentations of VDD include lethargy, increased vulnerability to bacterial and viral infections, exacerbation of existing chronic illness, the difficulty to lose weight and visceral obesity, insulin resistance, low backache, proximal muscle weakness muscle pain and spontaneous throbbing bone pain. Painful skeletal sites at times can be identified as pseudo-fractures using routine plain x-rays [9]. Groups at risk of VDD include Persons with limited sun exposure, elderly, Breastfed infants, individuals with dark skin, Persons with fat malabsorption, obese Persons or those who had a gastric bypass surgery [10].

Complementary VD supplements is important and it is more effective at increasing and maintaining the VD level in blood in conditions where it is difficult to provide adequate amounts of VD through exposure to solar radiation and dietary sources [11].

It has been shown that a combination of VD supplements with calcium can prevent hip and non-vertebral fractures in old men and women [12]. There is a suggestion that all adults with VDD to be managed with 50,000 IU of VD2 or VD3 once weekly for eight weeks or 6000 IU of VD2 or VD3 once per day to maintain serum level of VD more than $30 \mathrm{ng} / \mathrm{ml}$, then continued by maintenance treatment of 1500-2000 IU per day[2]. Recommendation for retesting VD level during 3 mo after starting supplementation [13]. The knowledge means what people understand of any given subject and the practice means how the people behave, in another meaning its refers to the habits in which they demonstrate their knowledge through their behaviors [14].

\section{Objectives of the study}

1) To determine the magnitude of knowledge and practices towards Vitamin D deficiency among women.

2) To identify the socio-demographic factors associated with knowledge and practices.

\section{MATERIALS AND METHODS}

Study design, setting and time: A cross-sectional study was carried out at consulting clinics of Imam Hussein Medical City in Karbala. Data collection was done between March 2 and June 30_2018.

Sampling method: A purposive sample of 442 women were approached (clients and companion of clients) who attended consulting clinics of Imam Hussein Medical City in Karbala.

Data collection procedure: Data was collected through a direct interview with women; the time needed for each interview was between 5 and $15 \mathrm{~min}$. 
Inclusion Criteria: The inclusion criteria include: females aged $18 \mathrm{y}$ old and over who agree to participate.

Exclusion criteria: Very ill and mentally retarded women, not previously diagnosed with VDD.

Subjects who were previously diagnosed with VDD assumed to have a high knowledge concerning VDD that's why the exclusion of them was done and this would shift the findings into a biased higher knowledge and practice regarding VD and its deficiency. Subjects who had a previous diagnosis of VDD were also excluded in the studies by (Salmanpour et al. 2016) and (Alemu and Varnam 2012) $[11,16]$.

Questionnaire form: A specially designed questionnaire (appendix) has been prepared; it was partially adapted from previous studies that assessing the knowledge and practices regarding VDD [15-21]. It was in English form then translated to Arabic one. It has been evaluated by specialists in community medicine and internal medicine.

The questionnaire consisted of 4 parts. The first part contains a single question; in which the subjects were asked whether they had a previous diagnosis with VDD or not. This point was for screening purpose, if their responses were yes; so they would be excluded from the study and continue with those who had no previous diagnosis with VDD.

The second part contains the socio-demographic data like age, marital status, residency, educational level and occupation.

The third part of the questionnaire consists of 8 knowledge related questions (mostly multiple choices questions) that included: the presence of knowledge about VD or not, if they had no knowledge, they would leave the questionnaire and their level of knowledge would be considered as poor.

Then the questionnaire had been continued with those who had knowledge about VD by asking them about other information like:

source of their knowledge, the main source of VD, food sources-rich in VD, its importance, its relation to calcium, problems associated with VDD and factors linked to its deficiency.

The fourth part was about practices related to VDD ( 5 close-ended questions; yes or no). The first question was about daily sun exposure; if yes: they would be continued to answer the duration of exposure and body parts that exposed to sun, but if the answer was no; they would be asked about the reasons for not being exposed to sunlight. Other questions were about the inclusion of VD rich foods to meals, the usage of sunscreen, usual intake of VD supplements and finally, they asked whether they had been checked their VD level before.

Statistical Analysis: Statistical Package for social sciences version 20 (SPSS v.20) was used for data input and analysis. Continuous variables presented as means and discrete variables presented as numbers and percentages. T-test, ANOVA and Pearson correlation were used to test the significance of observed variation in mean scores.

Findings with $P$ value of less than 0.05 were considered significant.

\section{RESULTS}

A total of 442 women aged $18 \mathrm{y}$ and above were approached and $430(97 \%)$ of them participated (The response rate). Fifty-eight $(13.5 \%)$ were excluded because they have a previous diagnosis of VDD, leaving $372(86.5 \%)$ responses for analysis.

The minimal age of the study subjects was $18 \mathrm{y}$ old, the maximum age was $71 \mathrm{y}$ old and the mean age was $41.16 \mathrm{y} \pm 13.15$ years. The majority of the participants had an aged range of 36-55. Most of the subjects $(81.5 \%)$ were housewives, small percent of them $(16.1 \%)$ were employee and $28 \%$ of them had been completed primary level.

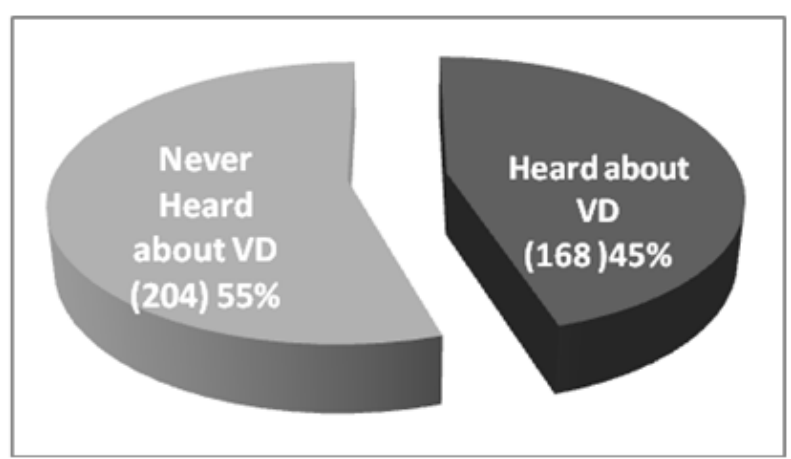

Fig. 1: Participants' knowledge about vitamin D

- The top two sources of participant knowledge were: friends/relatives followed by Health care providers. Sun was the most (72.6\%) source of VD that reported by the subjects.

- $\quad$ Less than half $(46.4 \%)$ of the subjects were knew that there is a relation between VD and calcium. Little percent (19\%) of the subjects had a good level of knowledge.

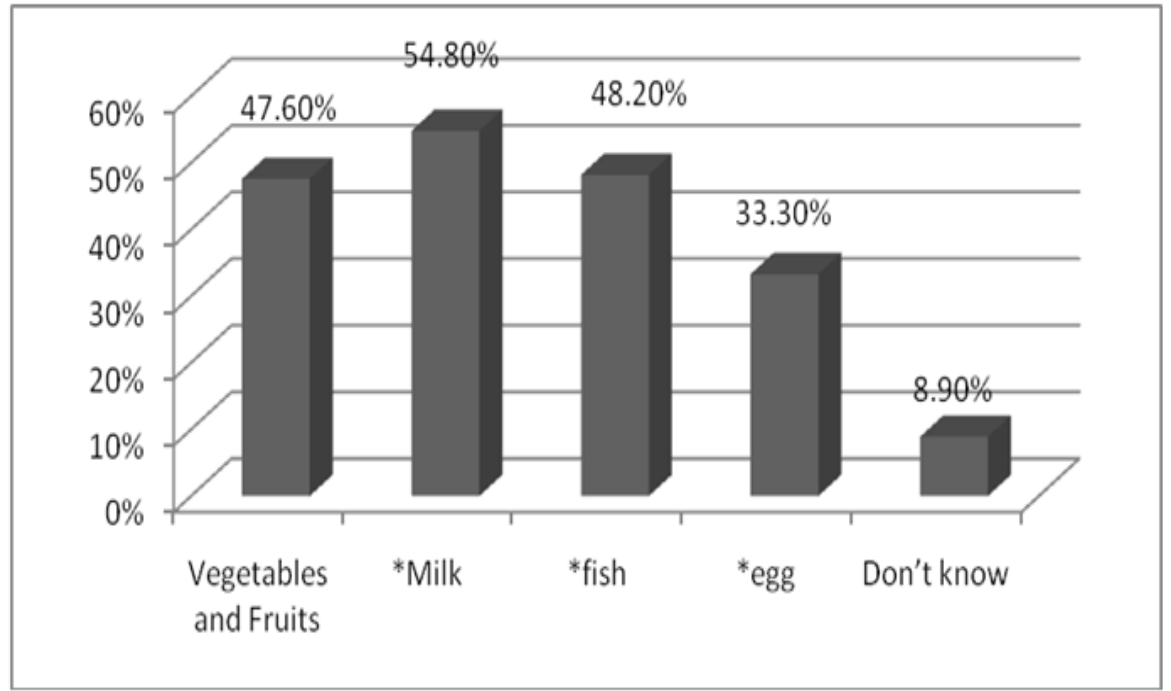

Fig. 2: Knowledge about food sources that contain VD ( $\mathrm{N}=168)$, asterisk $\left({ }^{*}\right)$ means the right response 


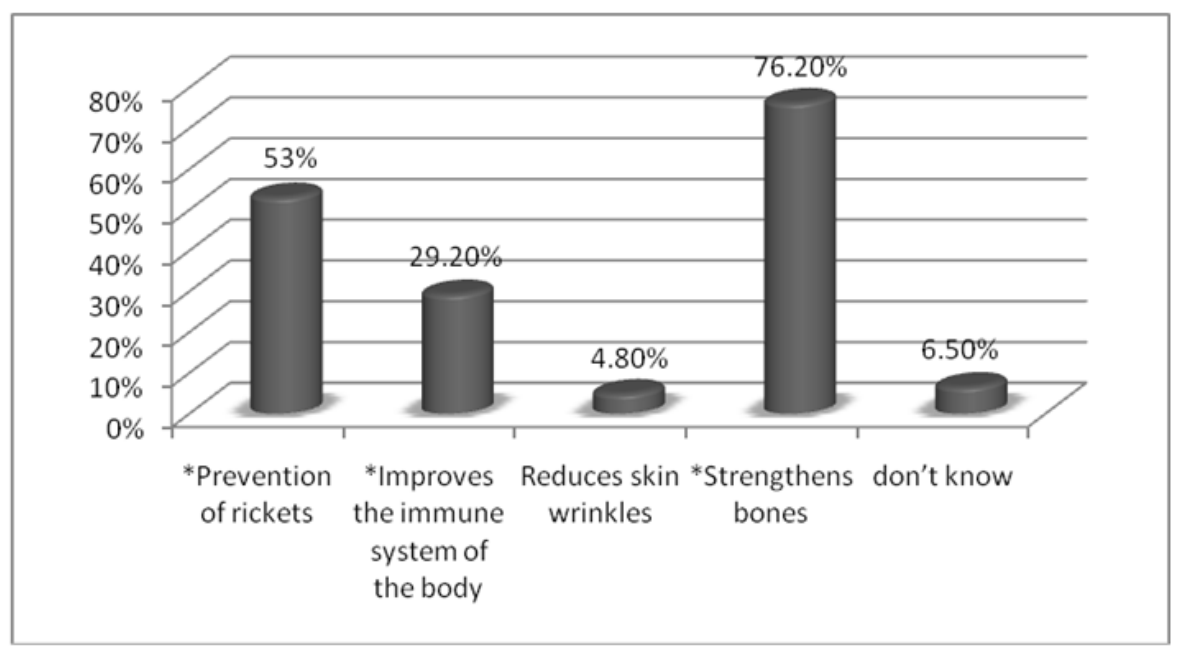

Fig. 3: Participants' knowledge about the importance of VD, asterisk (*) means the right response

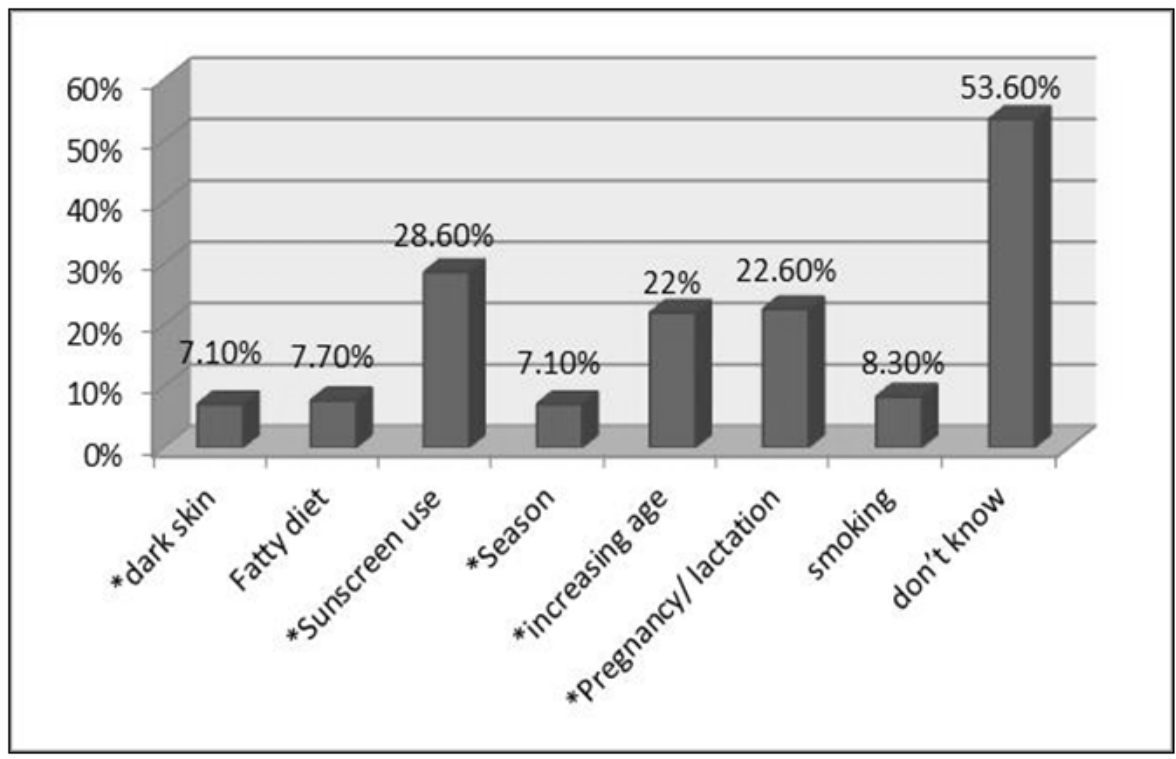

Fig. 4: Participants' knowledge about factors that decrease the amount of VD in the body ( $\mathrm{N}=168)$, (asterisk (*) means the right response)

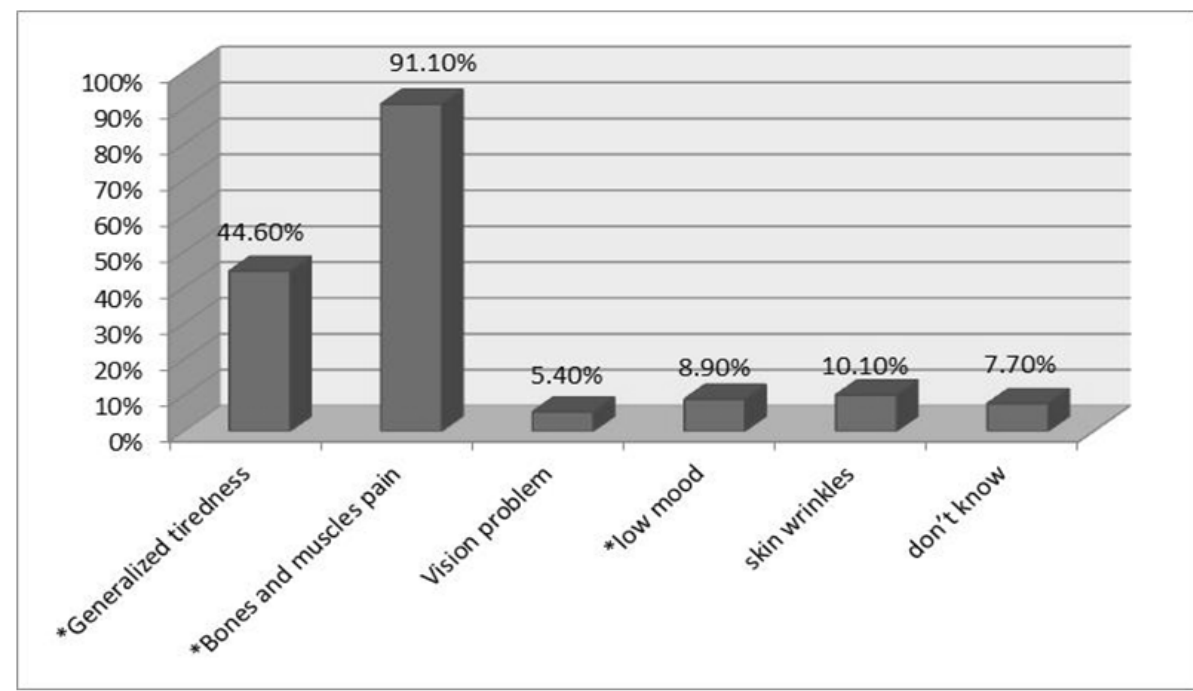

Fig. 5: Participants' knowledge about the problems associated with VDD ( $\mathrm{N}=168)$, (asterisk $(*)$ means the right response) 
Little percent (19\%) of the subjects had a good level of knowledge. The knowledge score is affected by level of education (those who completed Institute/college or higher having the higher knowledge score), there was a significant statistical association between them (P value<0.05)

Less than half $(48.2 \%)$ of the subjects had regular sun exposure. Most subjects $(85.7 \%)$ had been included foods rich in VD to their meal. Few numbers (8.9\%) were usually used sunscreen. Low percent $(22 \%)$ were usually taken VD supplements and a few numbers (6.5\%) had been tested their VD level before.

The more (51.9\%) reported the duration of daily sun exposure of the subjects was 10-20 min. Face and hands were the most parts that had been exposed to sun by the study subjects. The main reasons for being not exposed to sunlight that was reported in our study were indoor lifestyle and hot weather.

The mean score for the participants' practice about VDD was 2.53 out of $5(50.71 \%)$. Little percent (12\%) of the subjects had a good level of practice.

There is no statistical association between age, education, marital status or occupation and the mean practice score ( $\mathrm{P}$ value $>0.05$ ).

The increase in total knowledge was associated with an increase in the total practice score $(p=0.03)$.

\section{DISCUSSION}

Vitamin D is a very important element for women's health [22]. Our study provides a comprehensive view regarding the recent knowledge and practices towards VD among women attending the consulting clinics of Imam Hussein Medical City in Karbala. Unfortunately, less than half of the subjects (45.2\%)in the current study (who were not diagnosed with VDD) were learnt or heard about VD. Leaving $54.8 \%$ of the subjects who were not heard or learnt about it before completing the questionnaire. Our findings were nearly in line with the results by Oudshoorn et al. (2011) study, who studied the association between knowledge, VD status and dietary calcium intake in older people (mostly were females) in the Netherlands, It was found that $38 \%$ of the subjects had heard about VD [23]. While health care providers were not reported as a most popular source of knowledge in this study. A little visits by the study subjects to health institutions including primary health care except for ill health not to maintain health could be contributed to that results. Interestingly, most $(72.6 \%)$ of the subjects had been identified the sun as a main source of VD like what was reported by Arora et al.2016 study, who studied knowledge and practices of VD and attitude regarding sun among indian students [17]. While a small percent of the subjects were identified the sun as a main source of VD in Salmanpour et al. 2016 study, who studied knowledge and practices regarding VDD among the adult population in Sharjah, United Arab Emirates[15]. A higher percent of the subjects (54.8\%) were identified milk as good food sources of VD followed by fish (48.2\%) then egg (33.3\%). These results were in line with the findings by Babelghaith et al. 2017study, who studied knowledge and practice of VDD among people in Saudi Arabia [24]. On the other hand, a large percent $(47.6 \%)$ of the subjects had been identified incorrect food sources of VD (vegetables and fruits). This result was higher than a similar study of premenopausal women in Jeddah 2016 [25]. With regards to the importance of VD, most of the participants had knowledge about its role in bone health while a small percent were aware of its role in improving immune health. Our findings were better than what were mentioned in the studies by Salmanpour et al. 2016 and Kavitha 2015[15, 20]. Subjects in the current study were less knowledgeable regarding the relationship between VD and calcium than those in Khan et al. 2017 study [19].

A large percent $(53.6 \%)$ of the subjects in the current study were unaware about the factors that affect VD level. Also a high percent (65\%) of the subjects in Kavitha et al. 2015 study were didn't know the about the factors that affect VD level [20]. A considerable knowledge regarding the problems associated with VDD was existed among the subjects while a small percent of them were unaware about these problems.

Although a large percent of the subjects had identified the sun as a main source of VD, Less than half $(48.2 \%)$ had an average daily exposure to sunlight. While $51.8 \%$ of them did not expose to sunlight daily. Regarding to those who had no regular sun exposure; the main reasons that were reported in this study: indoor lifestyle and hot weather. Indoor lifestyle is a major predisposing factor for VDD in adults and children in most of the Middle East countries [26]. More than half (51.9\%) of the subjects (who had a regular sun exposure) exposed to sunlight at a period between 10 to $20 \mathrm{~min}$ per day.

Although the duration for sun exposure to get enough VD varies depending on the time of day, skin type, season and altitude [27], there is an evidence about the recommended time for VD production without skin burn; 10 to $30 \mathrm{~min}$ in the sun at time between 11:00 a. $\mathrm{m}$. and 3:00 p. m. Without the usage of sunblock, on average threefive times per week at the appropriate season [28]. Regarding body parts that exposed to the sun, the face and hands were the most exposed parts by the study subjects, while little percent of them exposing their long bones to the sun. Religious rules related to the type of clothing worn and cultural norms can be attributed to that behavior in our society.

Majority (85.7\%) of the study subjects include VD rich foods such as milk, fish or eggs to their meals, similar findings in Alemu and Varnam 2012 study[16]. Most (91.1\%) of the study subjects did not use sunscreen. This result might be attributed to the cost of the sunscreen or poor knowledge about sunscreen as a protective method against adverse effects of sun exposure. The present study showed a low percent (22\%) of the subjects taking VD supplements. National Institute of health recommends VD supplements to meet the daily requirement of VD for household individuals, females who wear long dress and head coverings for religious purposes, and individuals with occupations that limit sun exposure [29].

Although the subjects who had previous diagnoses of VDD were excluded from the study, the majority (93.5\%) of the subjects had never done VD test previously. This issue may reflect poor awareness of the study subjects concerning the importance of testing VD level. Also VD test is not available in the general hospitals and the expensive price of the test in the private hospitals and laboratories may contribute to that issue.

The current study showed that a small percent of the subjects had good level of knowledge and practices regarding VD with a positive significant correlation between knowledge and practice scores. This was similar to the result by Habib et al. study 2014 [18].

In conclusion, improving practices towards VD is directly related to increase knowledge about it.

\section{ACKNOWLEDGMENT}

A special feeling of gratitude to the mother of the corresponding author Mr. Maha Kashif Al-Ghataa, Who helps us in data entering and the writing language of this research.

Special thanks to the head of postgraduate studies in family medicine in Karbala University-College of medicine: Dr. Ali Abdulridha Kadhim Abutiheen for his unlimited support all over the times.

\section{AUTHORS CONTRIBUTIONS}

All the author have contributed equally.

\section{CONFLICT OF INTERESTS}

There is no possible conflict of interest related to this article was reported.

\section{REFERENCES}

1. Sallam MI, Abdulmajid ZT, Alamri AM, Gamal M, Gouda AM. Prevalence of vitamin D deficiency and community awareness among women's in Jeddah; 2017. p. 2.

2. Holick MF, Binkley NC, Bischoff-Ferrari HA, Gordon CM, Hanley DA, Heaney RP, et al. Evaluation, treatment, and prevention of vitamin d deficiency: an endocrine society clinical practice guideline. J Clin Endocrinol Metab 2011;96:1911-30.

3. Deschasaux M, Souberbielle JC, Partula V, Lecuyer L, Gonzalez $\mathrm{R}$, Srour B, et al. What do people know and believe about vitamin D? Nutrients 2016;8:718. 
4. Wacker M, Holick MF. Sunlight and vitamin D: a global perspective for health. Dermato Endocrinol 2013;5:51-108.

5. Pludowski P, Holick MF, Grant WB, Konstantynowicz J, Mascarenhas MR, Haq A, et al. Vitamin D supplementation guidelines. J Steroid Biochem Mol Biol 2018;175:125-35.

6. Webb A, Kift R, Durkin M, O'brien S, Vail A, Berry J, et al. The role of sunlight exposure in determining the vitamin D status of the UK white adult population. Br J Dermatol 2010;163:1050-5.

7. Norman AW, Bouillon R. Vitamin D nutritional policy needs a vision for the future. Exp Biol Med 2010;235:1034-45

8. Malaeb D, Hallit S, Salameh P. Assessment of vitamin D levels, awareness among lebanese pharmacy students, and impact of pharmacist counseling. J Epidemiol Global Health 2017;7:55-62.

9. Wimalawansa SJ. Non-musculoskeletal benefits of vitamin D. Steroid Biochem Mol Biol 2018;175:60-81.

10. Nair R, Maseeh A. Vitamin D: the "sunshine" vitamin. J Pharmacol Pharmacother 2012;3:118-26.

11. Zielinska Dabkowska K. Vitamin D. The truth about Vitamin D and sun exposure demystified. Finding the balance for personal health; 2014. p. 40-8.

12. Chen TC, Chimeh F, Lu Z, Mathieu J, Person KS, Zhang A, et al. Factors that influence the cutaneous synthesis and dietary sources of vitamin D. Arch Biochem Biophys 2007;460:213-7.

13. Taymouri MDF. Vitamin D in clinical practice; 2013.

14. Kaliyaperumal K. Guideline for conducting a knowledge, attitude and practice (KAP) study. AECS Illumination 2004;4:7-

15. Salmanpour VA, Ibrahim HS, Salameh AG, Yahya AM, Debal BK. Vitamin D deficiency: knowledge and practices among the adult population in Sharjah, United Arab Emirates. Arch Osteoporosis 2016;11:15.

16. Alemu E, Varnam R. Awareness of vitamin D deficiency among at-risk patients. BMC Res Notes 2012;5:17.

17. Arora H, Dixit V, Srivastava N. Evaluation of knowledge, practices of vitamin $d$ and attitude toward sunlight among Indian students. Evaluation 2016;9:308-13.
18. Habib FM, Al-Motairi WA, Al-Mutairi WM. Vitamin D deficiency: knowledge and practice among adult Saudi females. Glo Adv Res J Med Sci 2014;3:95-101.

19. Khan N. Attitudes and behavior towards sunlight exposure and knowledge about vitamin d among omani female university students. EC Nutr 2017;8:35-42.

20. Kavitha D. Knowledge, attitude and practice regarding vitamin d deficiency among antenatal mothers in tamilnadu. Int J Pharm Bio Sci 2015;6:486-97.

21. Boland S. A baseline assessment of university students' vitamin D knowledge; 2013.

22. Khadilkar SS. The emerging role of vitamin D3 in women's health. J Obstetrics Gynecol India 2013;63:147-50.

23. Oudshoorn C, A Hartholt K, Van Leeuwen J, Colin E, Velde N, Van der Cammen T. Better knowledge on vitamin D and calcium in older people is associated with a higher serum vitamin $\mathrm{D}$ level and a higher daily dietary calcium intake. Health Educ J 2012;71:474-82.

24. Babelghaith SD, Wajid S, Al-Zaaqi MA, Al-Malki AS, Al-Amri FD, Alfadly $\mathrm{S}$, et al. Knowledge and practice of vitamin D deficiency among people lives in Riyadh, Saudi Arabia-A cross-sectional study. Biomed Res 2017;28:3114-8.

25. Zareef T. Vitamin d status in relation to dietary intake, sun exposure obesity, lifestyle factors and bone health among saudi premenopausal women living in jeddah city, PhD Thesis; 2016.

26. Holick MF. The vitamin D epidemic and its health consequences. J Nutr 2005;135:2739s-48s.

27. Al-Kindi MK. Vitamin D status in healthy omani women of childbearing age: study of female staff at the royal hospital, muscat, oman. Sultan Qaboos University Med J 2011;11:56.

28. Christides T. Older Adults' Beliefs, knowledge and preferences for achieving healthy vitamin d status: a narrative review. Geriatrics 2018;3:26.

29. National Institution of Health. Vitamin D fact sheet for health professionals; 2018. Available from: https:// www.mayoclinicproceedings.org/article/S0025-6196601900/fulltext [Last accessed on 05 Jul 2019]. 